\title{
Adaptive Beamforming Algorithms for Smart Antenna System
}

\author{
Shun Na, Mengdi Guo, Yang Liu, Yinghui Zhang* \\ College of Electronic Information Engineering, Inner Mongolia University, Hohhot, 010021, China \\ ${ }^{*}$ Corresponding author: \\ E-mail address: yangliuimu@163.com.
}

Keywords: adaptive beamforming, smart antenna, MIMO.

\begin{abstract}
Adaptive beamforming based on specific needs to select the appropriate criteria or algorithms by changing the weight vector of antenna elements, so the signal through the right to receive the value of accumulated output signal corresponding to the optimum. We study the classical adaptive beamforming algorithms LMS and RLS for smart antenna multiple-inputmultiple-output (SA-MIMO) system, considering the different convergence factors and forgetting factors. We verify our analytical results and compare the performance of the adaptive beamforming for the different relevant parameters through Monte Carlo simulations.
\end{abstract}

\section{Introduction}

With the demand increase of the capacity to mobile communication systems and scarce spectrum resources, smart antennas are used to resolve co-channel interference, multiple access interference, multipath fading and other issues, as a new application to airspace resource development [1].The future fifth generation $(5 \mathrm{G})$ mobile network is aiming to provide a significant capacity increase compared to any current cellular solutions. The demand for increased capacity in wireless networks motivated recent research toward wireless systems that exploit space selectivity. A smart antenna consists of several antenna elements, whose signal is processed adaptively in order to exploit the spatial domain of the mobile radio channel.

The smart antenna technology can significantly improve wireless system performance and economics for a range of potential users. It enables operators of cellular and wireless local loop networks to realize significant increase in signal quality, network capacity and coverage. Smart antennas have been widely applied in radar, sonar, mobile and satellite communication [2], which inhibit the interference from different directions by beamforming and efficiently improve cell coverage and system capacity. Smart antenna has played a part in the 3rd Generation of Mobile Communication Systems (3G) standard, and the technique can also find applications in the next generation (5G) cellular systems [3], [4].Many researchers have been designed. For example, research on antenna design has focused in the selection of attractive radiating elements and antenna architecture.

Beamforming is a signal processing technique used in sensor arrays for directional signal transmission or reception. Adaptive beamforming is techniques in which arrays of antennas are used to achieve maximum reception in the direction of desired user while signals of same frequency form other directions are rejected. The user signal is multiplied by complex weights that adjust the magnitude and phase and amplitudes are adjusted to optimize the received signal. This causes the output of arrays of antenna to form transmit or receive in a particular direction and minimize the output in other direction. To change the directionality of the array when transmitting, a beamformer controls the phase and relative amplitude of the signal at each transmitter, in order to create a pattern of constructive and destructive interference in the wave front.

In this paper, adaptive beamforming algorithms LMS and RLS operation in MIMO smart antennas system is proposed. Moreover different convergence factors are used for the adaptive beamforming algorithms, and forgetting factors are also applied to each algorithm. 


\section{Adaptive beamforming algorithms}

For the last several years, adaptive beamforming plays an important role in sensor array systems in countering interference outside of the direction of interest. However, calculation of the adaptive weights generally requires a large number of operations that rapidly grows with the number of antennas. Consequently, a large number of programmable processors is commonly required to calculate the weights, which in some systems may present excessive weight, volume and power requirements. An algorithm is presented for the detection of users in the smart antenna system, which applied minimum bit error rate beamforming with interference cancellation at the BS side to enhance the capacity [5].

In SA-MIMO system, each antenna of MIMO is replaced by a smart antenna array. The inner product correlation matrix is dealt with a modified mean square error detection scenario based ordering successive interference cancellation [6]. In the 5G, an appropriate beamforming scheme to focus the transmitted and received signal in a desired direction in order to overcome the unfavorable path loss in one of the key enablers for cellular communications at mm Wave frequency bands [7]. The hybrid beamforming architecture in $\mathrm{mm}$ Wave bands are presented in [8], [9].In order to support this new paradigm shift in mobile communication radically new solutions for the air interface need to be developed. One of the critical challenges that the mobile industry will face is inter-cell interference due to the expected significant cell densification in order to support the required 10-fold increase in spectral efficiency. Beamforming algorithm based on the beam reference signal may include the formation of signal, DOA and the blind beamforming.

i) Based on the beam reference signal

Transmitting end of the transmission of such method requires known training sequence or pilot sequence, but without the need to determine or estimate the DOA of users. The more classical algorithm is LMS, RLS, direct matrix inversion and so on. Such algorithm is Robust, the performance will be better if the system synchronization under relatively ideal situation.

ii) Based on DOA beamforming

Algorithm determines such information without the sender of the reference signal, but requires determining DOA estimation techniques. The estimated by the DOA and its components corresponding weight value adjusting portion. Classic DOA estimated is MUSIC, ESPRIT and a series of improved algorithm based on two algorithms [10], [11].

iii) Blind beamforming

Blind algorithm obtain beam pattern to meet the requirements by adjusting the blind beamforming array antenna weighting vector. It does not need to send a known training sequence, and can be completed on the structural characteristics of the estimation of target users and testing using the received signal. The features include tamper model, non-Gaussian, stationary cycling and finite set of symbols [12], [13]. This paper focuses on two classical adaptive algorithms based on the reference signal

\subsection{LMS algorithm}

LMS algorithm use the instantaneous value of the gradient vector estimation method based on MMSE criterion and the steepest descent, using the stochastic gradient descent ideas, and ultimately achieve the purpose of minimizing the cost function. So the corresponding cost of using the instantaneous squared error.

The value of the right at time ${ }^{n}$ is noted by $w(n)$. According to the steepest descent algorithm, we can be drawn from $n+1$ right time to value:

$$
w(n+1)=w(n)-u \nabla_{w} \xi(n)
$$

where $\xi(n)=E\left\{\left.e(n)\right|^{2}\right\}$ is the mean square error performance function, $\nabla_{w} \xi(n)$ is the gradient of ${ }^{\xi}$.Convergence step factor noted $u$.

The error signal $e(n)$ is the error between the desired and actual output: 


$$
\begin{gathered}
e(n)=d(n)-w^{H}(n) x(n) \\
\nabla_{w} \xi(n)=2 R_{x x} w(n)-2 R_{x d}
\end{gathered}
$$

Because the steepest descent algorithm needs to know the exact value of $\nabla_{w} \xi(n), d(n)$ and $x(n)$ is required to receive a stable signal and know the second order statistics. Reality research, it is often used in place of the output of the instantaneous error MSE gradient $\hat{\nabla}_{w} \xi(n)$.

$$
\hat{\nabla}_{w} \xi(n)=\nabla_{w}|e(n)|^{2}=-2 e^{*}(n) x(n)
$$

Therefore, according to the LMS recurrence formula:

$$
\begin{aligned}
& w(n+1)=w(n)-\frac{1}{2} u \nabla_{w} \xi(n) \\
& w(n+1)=w(n)+u e^{*}(n) x(n)
\end{aligned}
$$

where $u$ is steps. If $u$ is small, slow to find the optimal solution, that is, slow convergence; if it is too large, it will have a great noise, may cause the system to diverge. The study showed that the optimal range of steps $1 \leq u \leq \frac{1}{2 \lambda_{\max }}$, where $\lambda_{\max }$ is a maximum characteristic value of $R_{x x}$.

\subsection{RLS algorithm}

If the spread of eigen-values $R_{x x}$ is larger, LMS convergence is slow. RLS algorithm is better to solute this problem. In each iteration time, containing an expression $R_{x x}^{-1}(n)$ instead of step $u$.We can get the following weight update equation:

$$
\begin{array}{r}
w(n+1)=w(n)-q(n) x(n) e^{H}(n) \\
q(n)=\frac{R_{x x}^{-1}(n) x(n+1)}{m+x^{H}(n+1) R_{x x}^{-1}(n) x(n+1)}
\end{array}
$$

where $R_{x x}^{-1}$ update to:

$$
R_{x x}(n)=\sum_{k=0}^{n} m^{n-k} x(n) x^{H}(n)
$$

where $m$ is forgetting factor.

$$
R_{x x}(n)=\frac{1}{m}\left\{R_{x x}^{-1}(n-1)-\frac{R_{x x}^{-1}(n-1) x(n) x^{H}(n) R_{x x}^{-1}(n-1)}{m+x^{H}(n) R_{x x}^{-1}(n-1) x(n)}\right\}
$$

where $q(n-1)=\frac{R_{x x}^{-1}(n-1) x(n)}{m+x^{H}(n) R_{x x}^{-1}(n-1) x(n)}, R_{x x}^{-1}(0)=\frac{1}{\varepsilon} I$.

RLS algorithm for the weighted squared error $\sum_{k=0}^{n} m^{n-k}|e(n)|^{2}$ is minimized. RLS convergence is fast, RLS algorithm is an order of magnitude faster than the LMS algorithm at the high SNR, but the more computational complexity than the LMS, and convergence instability.

\section{Simulation results}

\subsection{LMS algorithm performance analysis}

The simulation and analyze of convergence factors and the impact on system performance. The array element spacing, number of linear array elements is uniform 8 . Wherein the useful signal directions, the direction of the interference signal, of course, you can also select any other point of view. In addition to set the desired signal and interference signal at SNR $15 \mathrm{~dB}$ are taken, the channel is presence of white Gaussian noise, the simulation used to take snapshots 2000. 


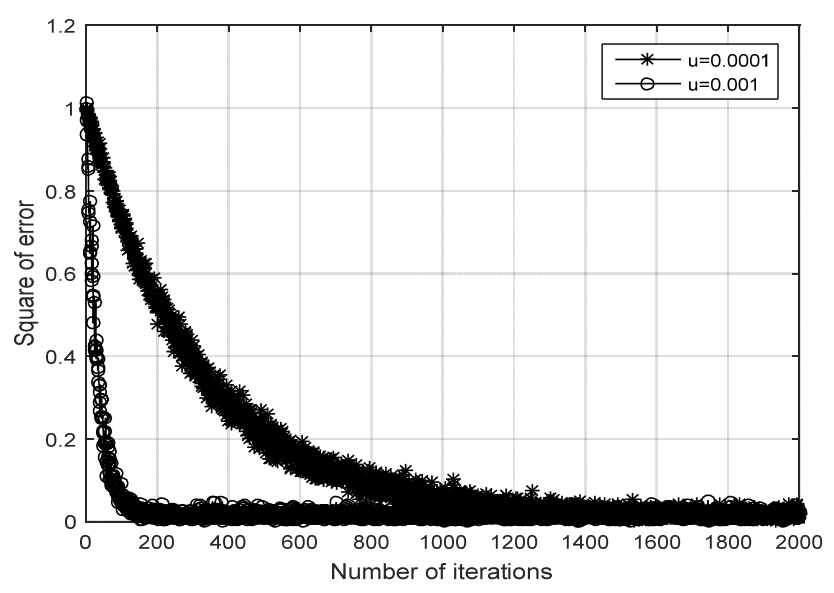

Fig.1: Steady-state error when different curves

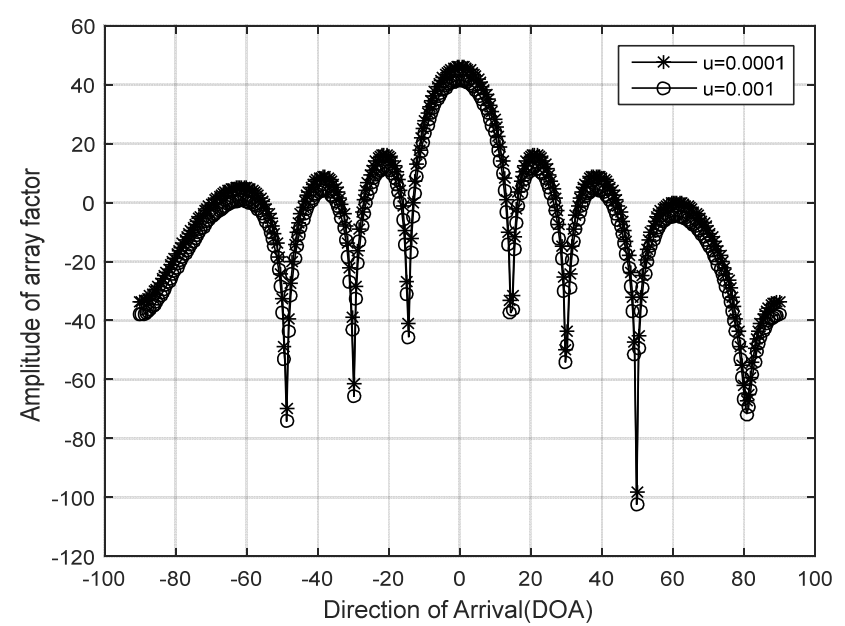

Fig.2: Cartesian coordinate system when different beam pattern

As shown in Fig.1, the steady-state error and the corresponding simulation obtained from observed when the step size selected, the corresponding algorithm requires an iterative curve almost 100 times reaches a plateau for $\mathrm{u}=0.001$. The step selection algorithm for $\mathrm{u}=0.0001$ corresponding need 1000 iterations to achieve the smooth curve. So we can know to choose the right step so that the algorithm can achieve convergence fast and effect.

In Fig.2, the Cartesian coordinate system and the corresponding beam pattern can be seen from the angle corresponding to the pattern shape is the same substantially, and it corresponds to the amplitude attenuation also very similar to that of the main beams are generated in the target user signal direction. The direction of the interference signal is formed sidelines or null also played a role in suppressing the interference signal.

\subsection{RLS algorithm performance analysis}

Observe and analyze the impact of forgetting factor on system performance. Elements spacing, the number of the array element to take uniform linear array 8 . Wherein the target user is DOA, and the corresponding user interference direction, and; snapshots of 2000; SNR is $15 \mathrm{~dB}$; zero mean and one variance of the Gaussian noise.

In Fig.3, we select different steady-state error forgetting factor, and curve obtained by simulation, which comparison forgetting factor taken. We can obtain the preliminary conclusion: despite influence of convergence rate about the choice of the forgetting factor is not very obviously, but when select a smaller forgetting factor, which the performance deteriorated significantly corresponds to the steady-state error. 


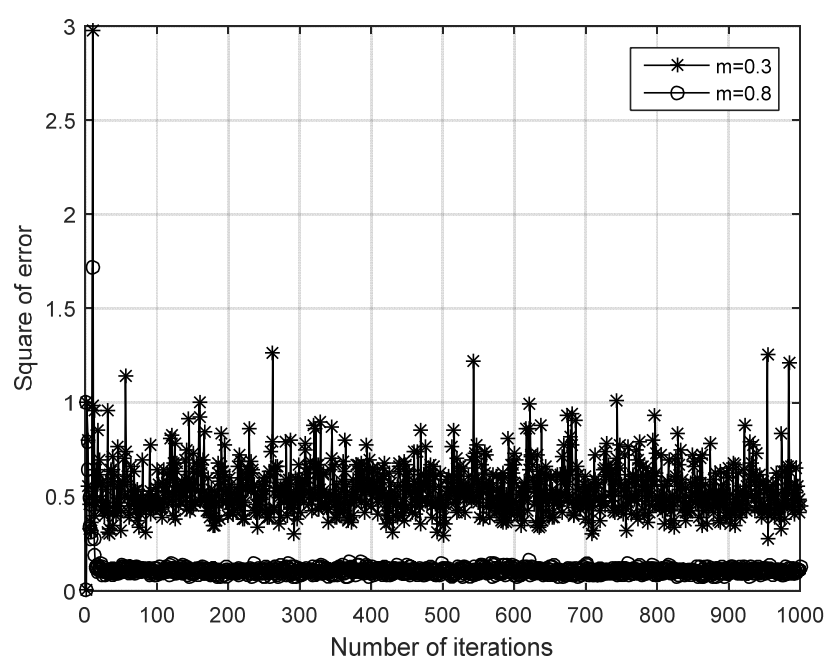

Fig.3: Steady-state error curves at different time m

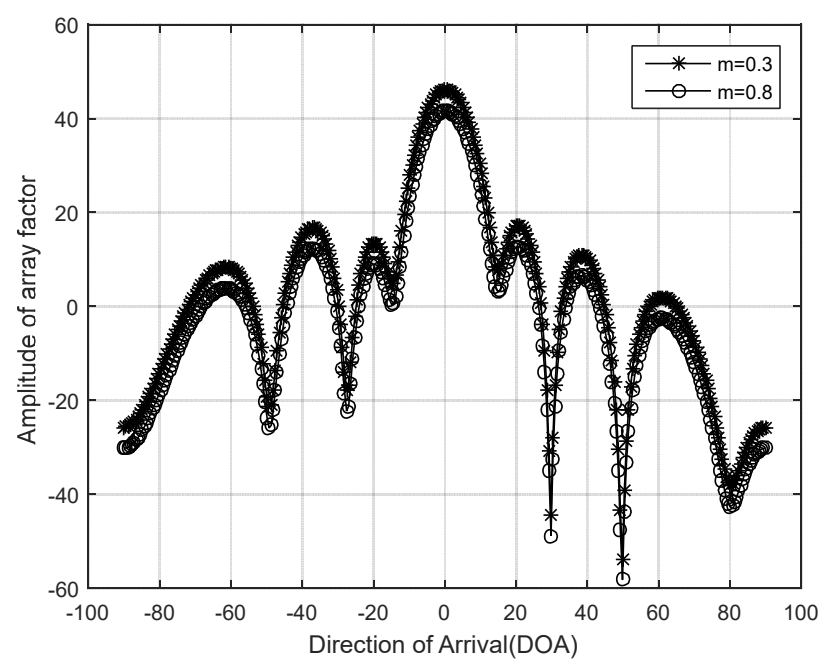

Fig.4: Cartesian coordinate different beam pattern $\mathrm{m}$

For the RLS algorithm, different Cartesian coordinate forgetting factor under the beam pattern seen from the Fig.4. If select a large forgetting factor, which will interfere with the user corresponding directions appear relatively deep nulls, which is the condition under the user interference suppression effect is relatively better.

\section{Conclusions}

The classic adaptive beamforming algorithms are described in detail and simulation analysis, including LMS and RLS. Simulation results show that when you select a larger step size, LMS algorithm steady-state error convergence curve relatively quickly. At the same time, choose a larger value of the steady-state error forgetting factor, RLS algorithm is relatively small and corresponding user interference in the direction of null is deep.

\section{Acknowledgements}

The authors are grateful to the National Science Foundation of China for its support of this research. This work is partly supported by the National Science Foundation of China under Grant 61362027 and 61461036, and the Natural Science Foundation of Inner Mongolia Autonomous Region of China under Grant 2016MS0604. 


\section{References}

[1] Zhang, H, Gao, L, Zhou L. HF super directive smart antenna system for interference suppression. IEICE Electron. Express, 12(3), pp. 1-7, 2015.

[2] Andersen, J.B. Array gain and capacity for known random channels with multiple element arrays at both ends. IEEE J. Sel. Areas Commun, 18(11), pp. 2172-2178, 2000.

[3] Roh, W, Yun, J, Jeongho, S. P, Millimeter-wave beamforming as an enabling technology for 5G cellular communications: theoretical feasibility and prototype results. IEEE Commun. Mag., 52(2), pp. 106-113, 2014.

[4] Rappaport, T. S, Millimeter wave Mobile Communications for 5G Cellular: It Will Work! IEEE Commun. Mag., 52(2), pp. 335-349, 2013.

[5] Bavand, M, Azmi, P, A Novel Interference Cancellation Aided Minimum Bit Error Rate Beamforming. 2011 Int. Sym. Computer Networks and Distributed Systems(CNDS), Tehran, Iran, pp. 35-39, 2011.

[6] Dai , J, J. Qi, Z. N, A suboptimal detection scheme of single-user SA-MIMO system, 2013 3rd Int. Conf. Consumer Electronics, Communications and Networks (CECNet), Xianning, China, pp.61-64, 2013.

[7] Pan, Z, Khan, F, An introduction to Millimeter-Wave Mobile Broadband Systems, IEEE Commun. Mag., 49(6), pp.101-107, 2011.

[8] Kim, T, Tens of Gbps Support with mm Wave Beamforming System for Next Generation Communications, IEEE GLOBECOM, pp. 3790-3795, 2013.

[9] Roh, W, Performances and Feasibility of $\mathrm{mm}$ Wave Beamforming System in Cellular Environments, IEEE ICC, pp. 2010-2016, 2013.

[10] Schmidt, R. O. Multiple emitter location and signal parameter estimation, IEEE Trans. Antennas and Propagation, 34(3), pp.276-280, 1986.

[11] Roy, R, Kailath, T. ESPRIT-A subspace rotation approach to estimation of parameters of cisoid in noise. IEEE Trans. Acoustics, Speech and Signal Processing, 34(5), pp.1340-1342, 1986.

[12] Liu, W. Blind adaptive wideband beamforming for circular arrays based on phase mode transformation, Digital Signal Processing, 21(2), pp. 239-247, 2011.

[13] Qin, B, Cai, Y. L. A low-complexity variable forgetting factor constant modulus RLS algorithm for blind adaptive beamforming, Signal Processing, 105(2), pp.277-282, 2014. 